\title{
Pelvic MRI, FDG-PET/CT, and Somatostatin Receptor Scintigraphy Findings of Treatment- Related Neuroendocrine-Differentiated Prostate Cancer
}

\author{
Kazuhiro Kitajima ${ }^{a}$ Shingo Yamamoto ${ }^{b}$ Masataka Ikedac

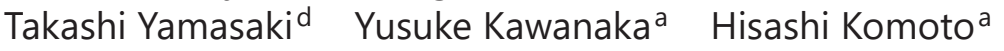 \\ Mitsunari Maruyama ${ }^{a} K_{\text {Kosuke Nishizaki }}{ }^{b}$ Kei Kimurac ${ }^{c}$ Neinei Kimurad \\ Koichiro Yamakadoa \\ aDepartment of Radiology, Hyogo College of Medicine, Hyogo, Japan; bepartment of \\ Urology, Hyogo College of Medicine, Hyogo, Japan; 'Division Lower GI, Department of \\ Surgery, Hyogo College of Medicine, Hyogo, Japan; dDepartment of Surgical Pathology, \\ Hyogo College of Medicine, Hyogo, Japan
}

\section{Keywords}

Neuroendocrine differentiation · Prostate cancer - MRI · FDG-PET/CT · Somatostatin receptor scintigraphy

\begin{abstract}
Treatment-related neuroendocrine-differentiated prostate cancer (NEPC) is a rare tumor entity that transdifferentiates from adenocarcinoma as an adaptive response to androgen receptor pathway inhibition. We report a 79-year-old male with treatment-related NEPC, presenting as rectal bleeding after hormonal therapy. MRI showed a $51 \times 52 \times 65 \mathrm{~mm}$ tumor occupying almost the whole prostate gland and invading the seminal vesicle and rectum as moderately heterogeneous hypointensity on T2-weighted image, restricted diffusion on apparent diffusion coefficient map and diffusion-weighted imaging, and heterogeneous enhancement on Gd-enhanced T1-weighted image. FDG-PET/CT showed strong FDG uptake of the prostate tumor, and somatostatin receptor scintigraphy (SRS) showed mild uptake of the prostate tumor. The surgically resected specimen revealed NEPC. If prostate cancer worsens despite conventional therapy, treatment-related NEPC should be considered, and the benefit of imaging examinations including prostate MRI, FDG-PET/CT, and SRS is in localizing lesions with neuroendocrine differentiation.




\section{Introduction}

Treatment-related neuroendocrine-differentiated prostate cancer (NEPC) is a lethal form of prostate cancer that emerges in the later stages of castration-resistant prostate cancer (CRPC) treatment. Treatment-related NEPC transdifferentiates from adenocarcinoma as an adaptive response to androgen receptor pathway inhibition $[1,2]$. The incidence of treatmentrelated NEPC has been rising due to the increasing use of potent androgen receptor pathway inhibitors. Typically, treatment-related NEPC is characterized by either low or absent androgen receptor expression, small-cell carcinoma morphology, and expression of neuroendocrine markers. Clinically, compared to conventional adenocarcinoma, treatment-related NEPC is characterized by an aggressive tumor biology with loss of prostate-specific antigen (PSA) secretion, unresponsiveness to androgen deprivation therapy, bulky tumor mass, development of bone or visceral metastases, and limited prognosis [1-4].

To our knowledge, there have been little reports discussing imaging characteristics of primary treatment-related NEPC. Here, we present prostate magnetic resonance imaging (MRI), ${ }^{18} \mathrm{~F}$-fluorodeoxyglucose positron emission tomography/computed tomography (FDG-PET/CT), and somatostatin receptor scintigraphy (SRS) with single photon emission computed tomography/computed tomography (SPECT/CT) of primary treatment-related NEPC proved pathologically by resected specimen.

\section{Case Report}

Three years ago, a 76-year-old man with biopsy-proven prostate cancer (Gleason score $4+5$ ), serum PSA level of $26.0 \mathrm{ng} / \mathrm{mL}$, and clinical stage of T3bNxM1b (lung metastasis was histopathologically diagnosed by CT-guided biopsy) underwent maximum androgen blockade therapy as initial treatment at another hospital. The PSA value reached the nadir of $0.036 \mathrm{ng} /$ $\mathrm{mL}$; however, the PSA value gradually increased to $0.244 \mathrm{ng} / \mathrm{mL}$. CRPC was diagnosed, and new hormonal therapy (enzalutamide) was started, which improved the PSA level to 0.003 $\mathrm{ng} / \mathrm{mL}$. However, rectal bleeding continued and the biopsy revealed neuroendocrine tumor (G3). For the operation, the patient was introduced to our hospital. Both neuron-specific enolase level and progastrin-releasing peptide level was high $(19.2 \mathrm{ng} / \mathrm{mL}$ and $576 \mathrm{pg} / \mathrm{mL}$, respectively). He underwent whole-body FDG-PET/CT, whole-body SRS using ${ }^{111}$ In-pentetreotide with SPECT/CT, and pelvic MRI. MRI demonstrated a $51 \times 52 \times 65 \mathrm{~mm}$ tumor occupying almost the whole prostate gland, invading the seminal vesicle and rectum. The illdefined tumor showed moderately heterogeneous hypointensity on T2-weighted image (Fig. 1a-c), hypointensity on T1-weighted image (Fig. 1d), restricted diffusion on diffusionweighted imaging (DWI) (Fig. 1e) and apparent diffusion coefficient map (Fig. 1f), and heterogeneous enhancement after intravenous administration of gadolinium diethylenetriaminepentaacetic acid (Gd-DTPA) (Fig. 1g, h). FDG-PET/CT scanning demonstrated strong FDG uptake (maximum standardized uptake value [SUVmax], 11.2) in the prostate tumor and no abnormal uptake in other sites (Fig. 2a, b). SRS scanning with SPECT/CT demonstrated mild uptake in the prostate tumor and no abnormal uptake in other sites (Fig. 2c, d). Although MRI showed a left pararectal lymph node measuring $7 \times 7 \mathrm{~mm}$, this small node showed no FDG uptake on FDG-PET/CT and no radiotracer uptake on SRS.

Because no distant metastatic lesion was observed on FDG-PET/CT and SRS with SPECT/ $\mathrm{CT}$, he underwent total pelvic exenteration including prostatectomy (Fig. 3a), pelvic lymphadenectomy, internal obturator muscle resection, and penile resection (pubic bone was also resected) in order to secure surgical margin. A pathologic examination revealed prostate small-cell carcinoma showing that the tumor cells are poorly differentiated, small to interme-

\section{Karger'}




\section{Case Reports in Oncology}

\begin{tabular}{l|l}
\hline Case Rep Oncol 2021;14:397-402 \\
\hline DOI: 10.1159/000511070 & $\begin{array}{l}\text { ○ 2021 The Author(s). Published by S. Karger AG, Basel } \\
\text { www.karger.com/cro }\end{array}$ \\
\hline
\end{tabular}

Kitajima et al.: MRI, FDG-PET, and SRS of Treatment-Related NEPC
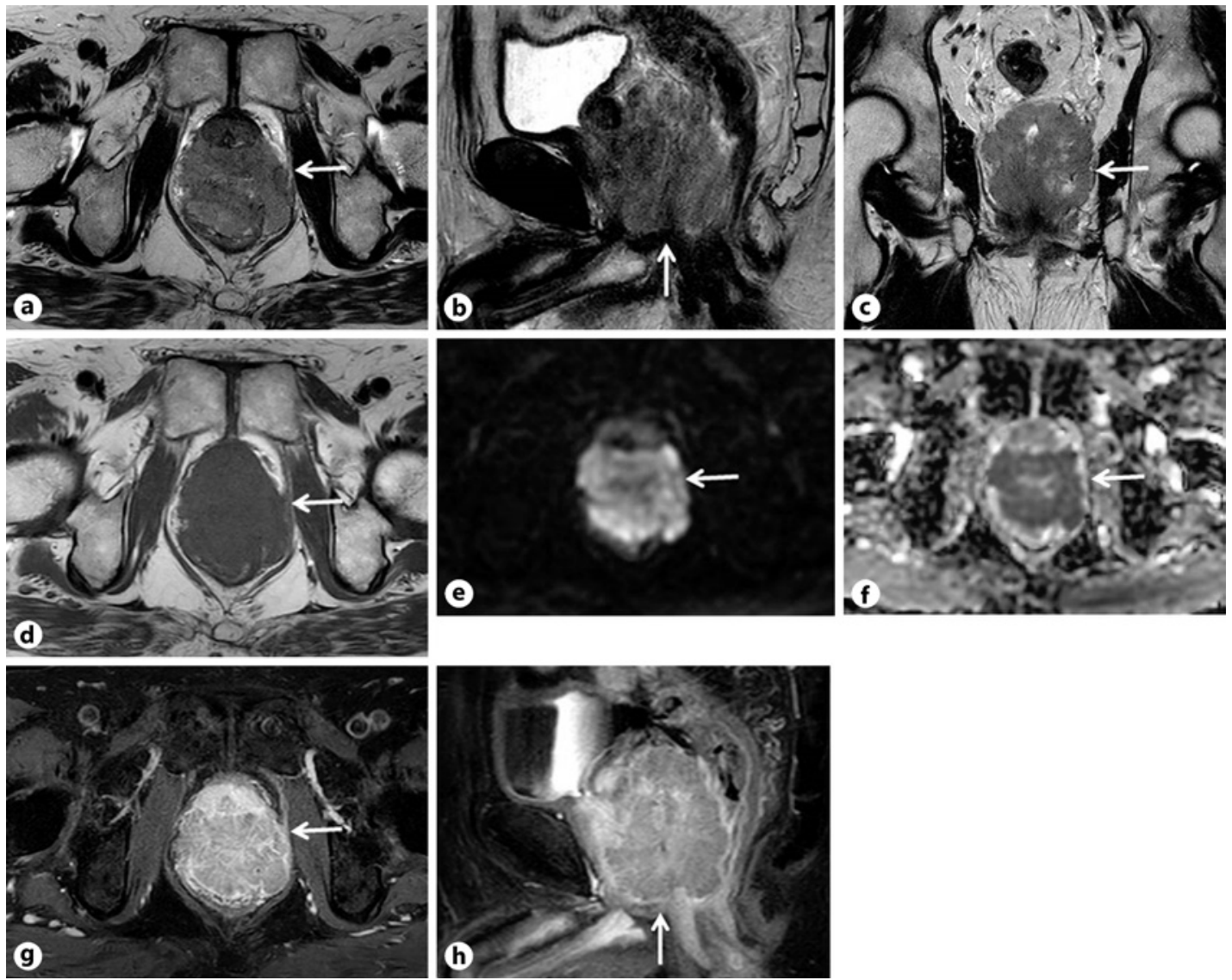

h

Fig. 1. Pelvic MRI. Axial (a), sagittal (b), and coronal (c) T2-weighted images show a $51 \times 52 \times 65$ mm tumor occupying almost the whole prostate gland and invading the seminal vesicle and the rectum as moderately heterogeneous hypointensity (arrow). d Axial T1-weighted image shows the prostate tumor as hypointensity (arrow). Axial diffusion-weighted image (e) and apparent diffusion coefficient map (f) show restricted diffusion in the mass (arrow). Axial (g) and sagittal (h) late-phase enhanced fat-suppressed T1-weighted images after Gd-DTPA administration show the heterogeneous enhancement of the prostate tumor invading the rectum (arrow).

diate in size, forming sheets and nests (Fig. $3 \mathrm{~b}$ ), and directly invading the seminal vesicle and rectal mucosa. The immunohistochemical findings with chromogranin $A$, synaptophysin, and CD56 were positive and Ki-67 index was 80\% (Fig. 3c-e). One pararectal lymph node metastasis was pathologically diagnosed.

\section{Discussion}

Treatment-related NEPC is a rare androgen-independent cancer subtype that develops at the later stage of CRPC. Treatment-related NEPC is an aggressive tumor entity with poor prognosis with survival ranging from 7 to 10 months [1-4]. Due to the rarity of the disease, the optimal treatment strategy is up to debate. Like in neuroendocrine cancers (NECs) of other organ systems, platinum-based chemotherapy regimens (cisplatin or carboplatin combined with etoposide) are commonly applied in first-line for advanced disease. In a literature review of 123 treatment-related NEPC patients, the time to development of treatment- 


\section{Case Reports in Oncology}

\begin{tabular}{l|l}
\hline Case Rep Oncol 2021;14:397-402 \\
\hline DOI: 10.1159/000511070 & $\begin{array}{l}\text { ○ 2021 The Author(s). Published by S. Karger AG, Basel } \\
\text { www.karger.com/cro }\end{array}$ \\
\hline
\end{tabular}

Kitajima et al.: MRI, FDG-PET, and SRS of Treatment-Related NEPC

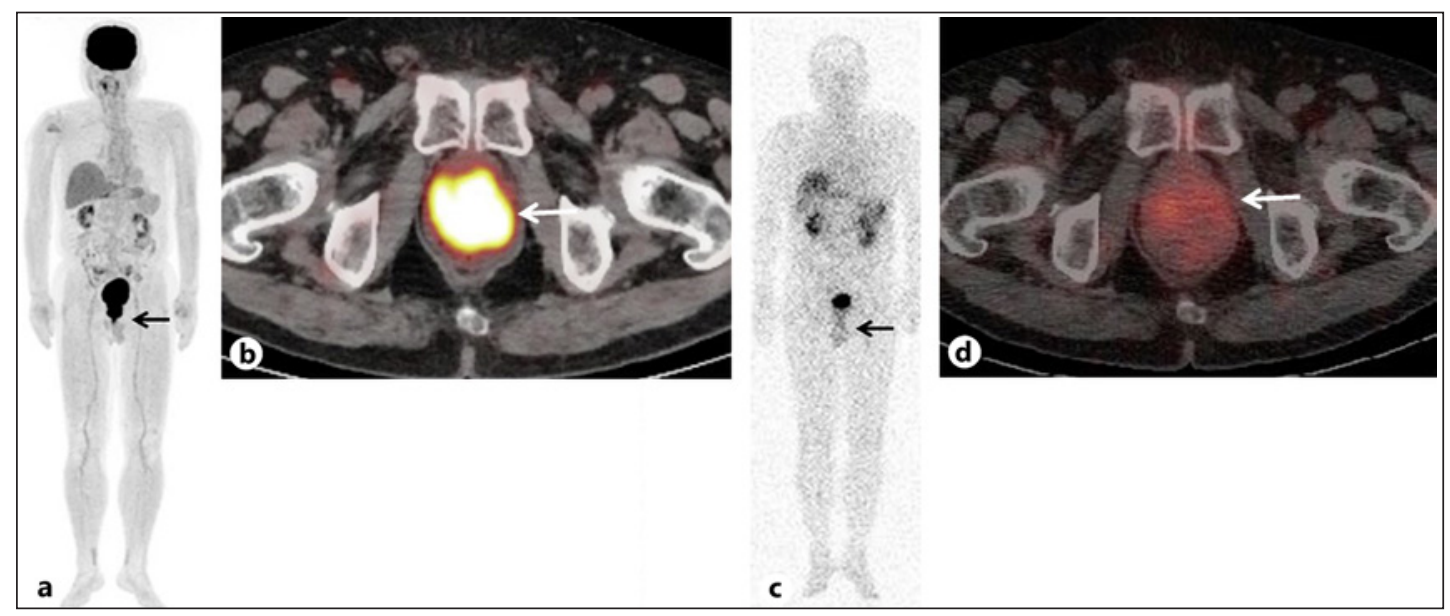

Fig. 2. FDG-PET/CT and SRS with SPECT/CT. a Maximum-intensity-projection (MIP) FDG-PET imaging shows strong FDG uptake in the prostate tumor (arrow). b Axial FDG-PET/CT shows strong FDG uptake (SUVmax, 11.2) of the prostate mass (arrow). c Whole-body SRS imaging shows mild uptake in the prostate mass (arrow). d Axial SRS with SPECT/CT shows mild uptake of the prostate mass (arrow).
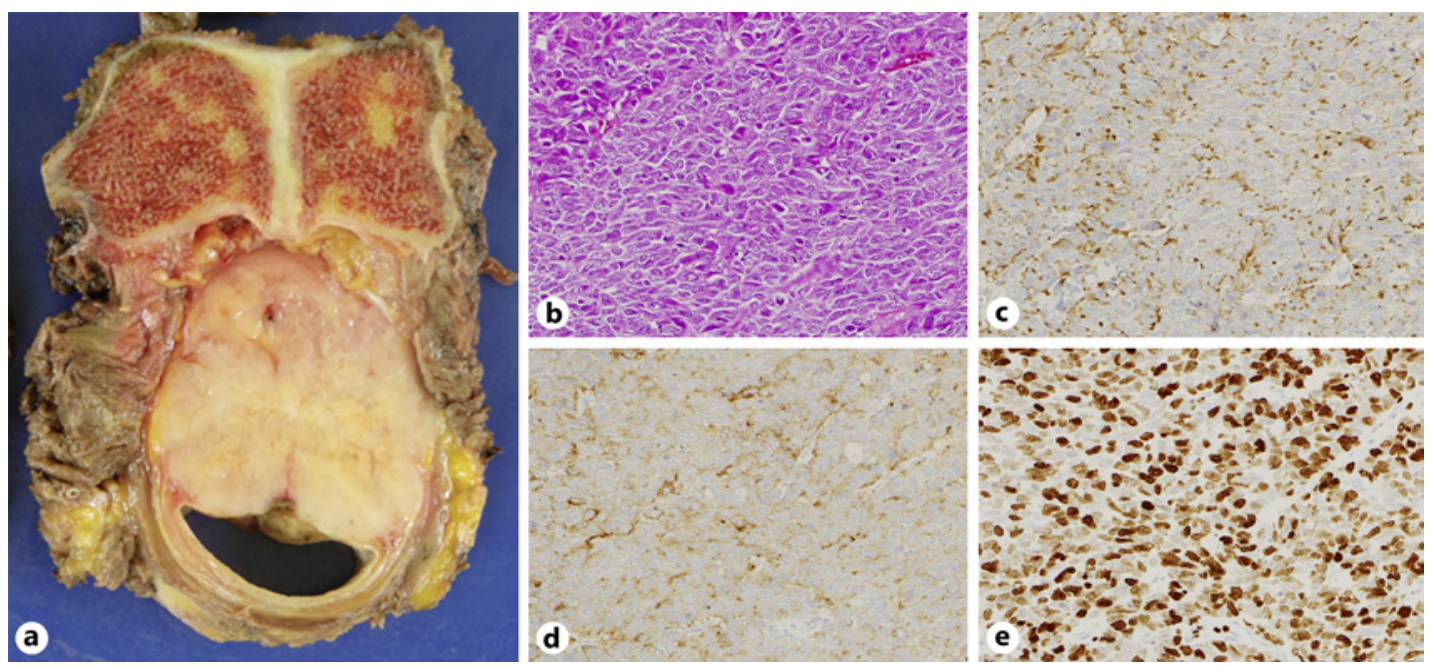

Fig. 3. Resected specimen and pathologic finding. a Resected specimen revealed a tumor occupying almost the whole prostate gland. $\mathbf{b}$ Hematoxylin and eosin-stained section of the small-cell carcinoma component showing that the tumor cells are poorly differentiated, small to intermediate in size, forming sheets and nests, and containing small, round hyperchromatic nuclei with indistinct nucleoli and scanty cytoplasm. The immunohistochemical findings with chromogranin A (c) and synaptophysin (d) were positive, and the Ki-67 index (e) was $80 \%$.

related NEPC from initial diagnosis of prostate cancer was 20 months, and the median survival after treatment-related NEPC was just 7 months [4]. Among the cases included, 56.3\% showed pure small-cell carcinoma histology, and $36.9 \%$ showed mixed adenocarcinoma and smallcell carcinoma histology. The duration of androgen deprivation therapy before transdifferentiation was $<24$ months in more than half of the patients. In a multivariate analysis, a high Gleason score $(\geq 8)$ was associated with a shorter time to treatment-related NEPC. 
To the best of our knowledge, only one paper has reported the MRI findings characteristics of 13 neuroendocrine prostate cancer patients in Chinese [5]. Of all 13 patients, three cases were primally diagnosed as small-cell cancer, and 10 cases were translated to neuroendocrine type from adenocarcinoma after endocrine therapy as well as our case. They demonstrated the neuroendocrine prostate cancer as moderately low signal intensity on T2WI, high signal on DWI, and peak and wash-out pattern on dynamic contrast-enhanced imaging after intravenous Gd-DTPA administration. The findings of T2WI and DWI are similar to our case. Bulky prostate tumor mass with these MRI findings regardless of low PSA is a clue to diagnose NEPC, and we suppose follow-up MRI is very useful.

Although FDG-PET/CT is not routinely recommended in common prostate adenocarcinoma due to low FDG uptake as compared with other malignancies (low glycolytic activity in prostate cancer cells), in general, FDG-PET/CT is useful to detect primary and metastatic NECs [6] and there have been reports demonstrating the utility of FDG-PET/CT in patients with NEPC $[7,8]$. In our case, primary NEPC showed strong FDG uptake, whereas unfortunately a tiny node metastasis was false-negative probably due to the limited spatial resolution of PET/CT machine.

By specifically targeting lesions with somatostatin receptors, SRS imaging might help to identify metastatic neuroendocrine tumor lesions, especially in types of G1 and G2 [9]. Kubota et al. [9] compared the uptake of SRS and FDG tracers in a total of 45 metastatic neuroendocrine tumor lesions in 15 patients and demonstrated that the uptake of the SRS and FDG tracers in neuroendocrine tumor lesions showed an inverse correlation. The G1+2 lesions had a higher SRS uptake than the G3 lesions, whereas the G1+2 lesions had a significantly lower FDG uptake than the G3 lesions. The uptake SRS of NECs is usually low. Another promising application of SRS imaging is its utility for visually evaluating the effects of therapy [10]. While changes in tumor markers help predict the overall effect of therapy, changes in the uptake detected by SRS imaging can demonstrate regional improvement. Thus, SRS imaging might provide information that conventional tumor markers or imaging studies cannot disclose.

\section{Conclusion}

When prostate cancer worsens despite conventional therapy, treatment-related NEPC should be considered. In general, while the clinical course and tumor markers help predict the differentiation of prostate cancer into treatment-related NEPC, the benefit of imaging examinations including prostate MRI, FDG-PET/CT, and SRS is in localizing lesions with neuroendocrine differentiation for early diagnosis and the introduction of the appropriate treatment.

\section{Statement of Ethics}

This report complies with the guidelines for human studies and includes evidence that the research was conducted ethically in accordance with the World Medical Association Declaration of Helsinki. The authors have no ethical conflicts to disclose. Written informed consent was obtained from the patient for publication of this case report and any accompanying images. 


\section{Conflict of Interest Statement}

The authors have no conflicts of interest to declare.

\section{Funding Sources}

This work was supported by JSPS KAKENHI grant number 19 K08187.

\section{Author Contributions}

Concept and design, K. Kitajima, S. Yamamoto, K. Yamakado; acquisition of data, S. Yamamoto, M. Ikeda, T. Yamasaki, Y. Kawanaka, H. Komoto, M. Maruyama, K. Nishizaki, K. Kimura, N. Kimura; drafting of the manuscript, K. Kitajima; critical revision of the manuscript for important intellectual content, S. Yamamoto, K. Yamakado. All authors approved final version of manuscript.

\section{References}

1 Akamatsu S, Inoue T, Ogawa O, Gleave ME. Clinical and molecular features of treatment-related neuroendocrine prostate cancer. Int J Urol. 2018;25:435-51.

2 Apostolidis L, Nientiedt C, Winkler EC, Berger AK, Kratochwil C, Kaiser A, et al. Clinical characteristics, treatment outcomes and potential novel therapeutic options for patients with neuroendocrine carcinoma of the prostate. Oncotarget. 2019;10(1):17-29.

3 Zaffuto E, Pompe R, Zanaty M, Bondarenko HD, Leyh-Bannurah SR, Moschini M, et al. Contemporary incidence and cancer control outcomes of primary neuroendocrine prostate cancer: A SEER Database Analysis. Clin Genitourin Cancer. 2017;15(5):e793-800.

4 Wang HT, Yao YH, Li BG, Tang Y, Chang JW, Zhang J. Neuroendocrine prostate cancer (NEPC) progressing from conventional prostatic adenocarcinoma: factors associated with time to development of NEPC and survival from NEPC diagnosis-a systematic review and pooled analysis. J Clin Oncol. 2014;32(30):3383-90.

5 Zhang WH, Si TG, Yang XL, Yang B, Liu CF, Yu HP, et al. [Clinical characteristics of 13 neuroendocrine prostate cancer patients]. Zhonghua Yi Xue Za Zhi. 2017;97(17):1316-9.

6 Panagiotidis E, Bomanji J. Role of 18F-fluorodeoxyglucose PET in the study of neuroendocrine tumors. PET Clin. 2014;9(1):43-55.

7 Spratt DE, Gavane S, Tarlinton L, Fareedy SB, Doran MG, Zelefsky MJ, et al. Utility of FDG-PET in clinical neuroendocrine prostate cancer. Prostate. 2014;74(11):1153-9.

8 Parida GK, Tripathy S, Datta Gupta S, Singhal A, Kumar R, Bal C, et al. Adenocarcinoma prostate with neuroendocrine differentiation: Potential utility of 18F-FDG PET/CT and 68Ga-DOTANOC PET/CT over 68Ga-PSMA PET/CT. Clin Nucl Med. 2018;43(4):248-9.

9 Kubota K, Okasaki M, Minamimoto R, Miyata Y, Morooka M, Nakajima K, et al. Lesion-based analysis of (18) F-FDG uptake and (111)In-Pentetreotide uptake by neuroendocrine tumors. Ann Nucl Med. 2014;28(10): 1004-10.

10 Mori H, Nakajima K, Kadomoto S, Mizokami A, Ikeda H, Wakabayashi H, et al. Imaging somatostatin receptor activity in neuroendocrine-differentiated prostate cancer. Intern Med. 2018;57(21):3123-8. 